\title{
Curbside Consult
}

\section{Exercise for cancer patients: a new challenge in sports medicine}

In the past, physicians usually advised patients with chronic diseases to rest and avoid physical effort. These recommendations were empirical: because most chronic diseases are associated with functional changes resulting in impaired physical performance, exercise in this group of patients may generate fatigue, breathlessness, and tachycardia. Therefore, avoiding physical activity results in less discomfort.

\section{Exercise could play a role}

\section{as complementary therapy}

\section{for cancer patients during}

\section{and after treatment}

In the past few years, however, scientific evidence has dramatically changed our ideas about exercise for patients with chronic diseases. In the late 1960s, the inclusion of physical activity in rehabilitation programs for patients who had had myocardial infarction set a milestone and opened up new perspectives for the use of exercise in the treatment of chronic diseases. It is now well established that excessive rest and lack of physical activity may result in severe deconditioning and, thus, reduce the functional status and quality of life of patients with chronic illness. Furthermore, numerous studies have shown that exercise is an effective means for counteracting several of the adverse effects that chronic diseases have on physical performance. As a result of this evidence, exercise is actually considered an essential component in the treatment of several illnesses, including peripheral vascular disorders, chronic obstructive lung disease, ischemic myocardial disease, and heart failure. The value of exercise for rehabilitation has been widely recog- nized; indeed, many institutions offer exercise programs for organ transplant recipients and for patients after myocardial infarction or with chronic renal failure. Until recently, however, less was known about the feasibility and effects of exercise programs for cancer patients during and after treatment.

The role of exercise in oncologic rehabilitation programs has thus far been mostly limited to physical treatment addressing specific impairments caused, for example, by amputation or surgery. However, the medical attitude regarding exercise for cancer patients is changing fast. The recent world class performances of athletes who have been treated for cancer have focused attention on the effects of training on the physical performance of cancer patients. Moreover, recent studies have shown that physical activity may improve the quality of life, mood, and the physical performance of cancer patients during and after treatment.

Regular physical activity has been shown to increase the performance status in breast cancer patients treated with conventional chemotherapy ${ }^{1}$ and in patients after bone marrow transplantation. ${ }^{2}$ It has also been shown to reduce psychological distress and fatigue in patients treated with radiotherapy ${ }^{3}$ and after high-dose chemotherapy with peripheral blood stem cell transplantation. ${ }^{4}$ Furthermore, a reduction of treatmentrelated complications has been observed in cancer patients participating in exercise programs during cancer treatment. ${ }^{5,6}$ Finally, preliminary evidence suggests that regular physical activity may improve immune function. $^{7-9}$ Therefore, exercise could play a role as complementary therapy for cancer patients during and after treatment.

More information is needed, however, about the effects and feasibility of exercise programs for different groups of patients with oncologic diseases. Indeed, "cancer" is a common denominator for more than 100 neoplastic diseases, each with a different cause, course, and prognosis. The natural history of the same nosologic disorder may vary considerably in different settings - that is, acute lymphoblastic leukemia in children and adults. Finally, cancer patients may have many specific problems. Chemotherapy can damage bone marrow and thereby impair the production of red blood cells; the resulting anemia decreases the oxygen transport capacity of the blood. Agents like anthracyclines and cyclophosphamide and irradiation of the mediastinum can result in myocardial damage and a decrease of cardiac output. Metastatic disease and pleural effusion cause a reduction of total lung capacity; furthermore, changes in the pulmonary architecture due to surgical treatment of primary or metastatic lung cancer or as sequelae to fibrosis after radiotherapy may alter the ventilation-toperfusion ratio. Treatment with immunosuppressive agents (for example, high-dose corticosteroids and cyclosporine) can lead to a marked loss of muscle mass and severe myopathy. Furthermore, reduced protein and calorie intake as a consequence of anorexia and nausea and impaired absorption after gastrointestinal surgery may lead to a negative nitrogen balance and, hence, to a catabolic state. Finally, an increase in the concentration of cytokines-interleukins 1 and 6, tumor necrosis factor, and alpha interferonresulting from the interaction between the tumor and the host-defense system has been

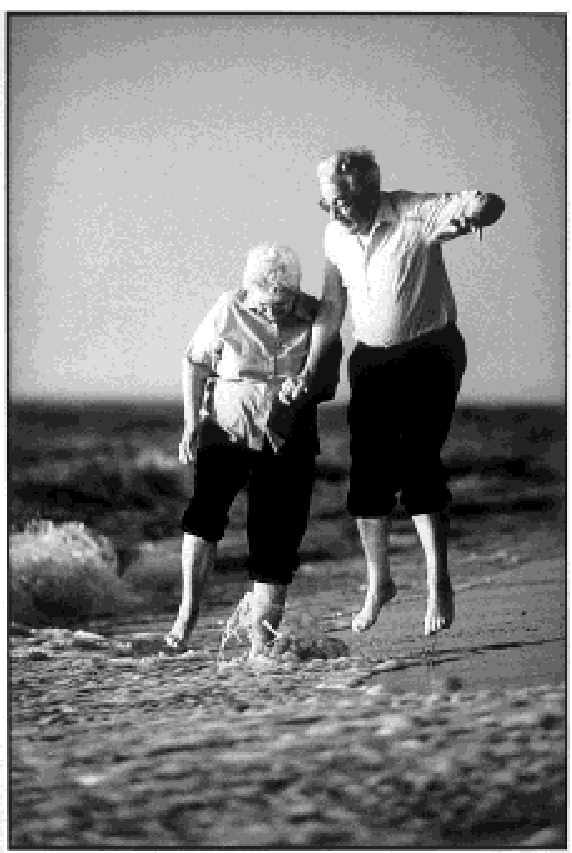


associated with muscular waning. All these factors may affect patients' physical condition and reduce their performance and must be carefully considered when designing an exercise program.

Research about the effects of exercise in the prevention and rehabilitation of cancer and of physical activity on immune function is still at its beginning. However, this is going to be one of the most active areas of research in sports medicine in the coming decade. It is certainly time to meet the challenge.

References

1 MacVicar MG, Winningham ML, Nickel JL. Effects of aerobic interval training on cancer patients' functional capacity. Nurs Res 1989;38:348-351.
2 Dimeo F, Bertz H, Finke J, Fetscher S, Mertelsmann R, Keul J. An aerobic exercise program for patients with haematological malignancies after bone marrow transplantation. Bone Marrow Transplant 1996;18:1157-1160.

3 Mock V, Dow KH, Meares CJ, et al. Effects of exercise on fatigue, physical functioning, and emotional distress during radiation therapy for breast cancer. Oncol Nurs Forum 1997;24:991-1000.

4 Dimeo FC, Stieglitz RD, Novelli-Fischer U, Fetscher $\mathrm{S}$, Keul J. Effects of physical activity on the fatigue and psychological status of cancer patients during chemotherapy. Cancer 1999;85:2273-2277.

5 Winningham ML, MacVicar MG, Bondoc M Anderson JI, Minton JP. Effect of aerobic exercise on body weight and composition in patients with breast cancer on adjuvant chemotherapy. Oncol Nurs Forum 1989;16:683-689.

6 Dimeo F, Fetscher S, Lange W, Mertelsmann R, Keul

$\mathrm{J}$. Effects of aerobic exercise on the physical

performance and incidence of treatment-related complications after high-dose chemotherapy. Blood 1997;90:3390-3394

7 Nehlsen-Cannarella SL, Nieman DC, Balk-Lamberton $\mathrm{AJ}$, et al. The effects of moderate exercise training on immune response. Med Sci Sports Exerc 1991;23:64-70.
8 Kaufman JC, Harris TJ, Higgins J, Maisel AS. Exercise-induced enhancement of immune function in the rat. Circulation 1994;90:525-532.

9 Crist DM, Mackinnon LT, Thompson RF, Atterbom HA, Egan PA. Physical exercise increases natural cellular-mediated tumor cytotoxicity in elderly women. Gerontology 1989;35:66-71.

Fernando Dimeo

Department of Sports Medicine Benjamin Franklin Medical Center Freie Universitaet Berlin

Clayallee 229

14195 Berlin, Germany

Correspondence to:

Dr Dimeo

ferdimeo@zedat.fu-bevlin.de

Competing interests: None declared

This article was published in $\mathrm{Br} J$ Sports Med 2000;34:160-161 